\title{
Linear growth and poly $(\beta$-hydroxybutyrate $)$ synthesis in response to pulse-wise addition of the growth-limiting substrate to steady-state heterotrophic continuous cultures of Aquaspirillum autotrophicum
}

\author{
Marco Pagni,* Trello Beffa, Cédric Isch and Michel Aragno \\ Laboratoire de Microbiologie de l'Université de Neuchâtel, Chantemerle 22, 2007 Neuchâtel, Switzerland
}

(Received 4 April 1991; revised 3 September 1991; accepted 31 October 1991)

\begin{abstract}
Heterotrophic pyruvate-limited steady-state continuous cultures of the bacterium Aquaspirillum autotrophicum were perturbed with a pulse injection of a small volume of concentrated pyruvate solution. These cultures exhibited an instantaneous change in the growth dynamics, turning from steady state to apparently linear growth. These transient growth-responses had no lag phase and were clearly distinct from unlimited exponential growth according to the initial rates of increase of biomass and substrate disappearance kinetics. A linear accumulation with time of poly ( $\beta$-hydroxybutyrate) was observed within the cells. Slopes of these linear responses were negatively correlated with the dilution rate. Physiological bases of linear growth are discussed in the light of the models of $\mathbf{H}$. $\mathbf{E}$. Kubitschek. Poly ( $\beta$-hydroxybutyrate) synthesis in the absence of exogenous limitation may serve to protect the cells against a transient metabolic overflow.
\end{abstract}

\section{Introduction}

Continuous culture is most often used to study steadystate growth physiology. However, only part of the regulatory characteristics of the metabolism are then expressed. Such studies cannot account for most of the growth responses in natural environments, which are essentially a succession of transitions. Several standard transitions can be studied with continuous cultures; a typical example is represented by the instantaneous suppression of the limitation by a pulse of the limiting substrate. Such experiments have been reported by Harisson \& Maitra (1969) with Klebsiella aerogenes and more recently by Brooke $e$ al. (1989) with a thermophilic Bacillus strain.

The facultatively autotrophic bacterium Aquaspirillum autotrophicum was chosen as model organism. Apart from its ability to grow autotrophically under $\mathrm{H}_{2} / \mathrm{O}_{2} / \mathrm{CO}_{2}$ mixtures, it can grow heterotrophically under air with a variety of organic acids (Aragno \& Schlegel, 1978a,b), as well as mixotrophically (Fasnacht, 1988). It is therefore a valuable organism to study various types of transitions with regard to energy and carbon metabolism. Moreover, it forms homogeneous suspen-

* Author for correspondence. Tel. 38 256434; fax 38 242695; email mbpagni@cnedcu51.bitnet.

Abbreviation: PHB, poly( $\beta$-hydroxybutyrate). sions in culture, and does not excrete polysaccharides. Poly( $\beta$-hydroxybutyrate), PHB, is synthesized as a reserve material under nitrogen limitation (WaltherMauruschat et al., 1977).

Pulse experiments similar to those described above were undertaken in two different types of continuous culture apparatus. One was designed for on-line turbidimetric measurements at short intervals $(6 \mathrm{~min})$, requiring relatively low biomass densities; this allowed a description of transient kinetics with satisfactory accuracy on a $1 \mathrm{~h}$ scale. The other apparatus was used to sample cultures for chemical analysis, thus needing higher biomass densities.

\section{Methods}

Organism and culture conditions. Aquaspirillum autotrophicum strain SA32 (DSM 732, ATCC 29984, Aragno \& Schlegel 1978a) was precultivated in the basal mineral medium described by Schlegel $e t$ al. (1961) and Aragno \& Schlegel (1981) with $0.5 \%$ sodium pyruvate added. The temperature was maintained at $27^{\circ} \mathrm{C}$ throughout the experiments.

For turbidimetric observation of growth kinetics, cells were grown in six 'home-made' fermenters running in parallel. Each fermenter had a working volume of $800 \mathrm{ml}$ and was stirred at 1500 r.p.m. with a large magnetic stirrer. The feed medium consisted of the basal medium with only half the phosphate concentration and with the addition of sodium pyruvate at a final concentration of $7.5 \mathrm{mM}$. For pulse experiments, 1 or $2 \mathrm{ml}$ sterile $3 \mathrm{M}$-sodium pyruvate was injected directly into the culture. 
The partial pressure of dissolved oxygen and the $\mathrm{pH}$ were followed with immersed electrodes. Feed medium and thermostatization were common to all fermenters; these differed only in their dilution rate and in their air inflow rate. Optical density at $436 \mathrm{~nm}$ was measured automatically every $6 \mathrm{~min}$ in each fermenter, with an accuracy of more than $1 \%$, with a PU8620 Philips spectrophotometer and a HELMA $170.004-\mathrm{QS}$ flow-cell with a $2 \mathrm{~mm}$ optical path.

For experiments in which samples had to be collected for chemical analyses, cells were grown in a Biostat-S fermenter with a working volume of 3.41 stirred at 800 r.p.m. Sodium pyruvate was added to the basal mineral medium at $22.26 \mathrm{~mm}$ final concentration. Sterile $3 \mathrm{M}$ sodium pyruvate $(15 \mathrm{ml})$ was injected for pulse experiments. Sampling was usually performed with an automatic collecter, but, during transitions, samples were collected in a large precooled glass beaker and continuously stirred on an ice bed until the temperature fell to $1{ }^{\circ} \mathrm{C}$ (less than $30 \mathrm{~s}$ ). Optical density was measured with a Zeiss PM4 photometer at $436 \mathrm{~nm}$ with an optical path of $1 \mathrm{~cm}$.

PHB determination. The PHB assay was a combination of the methods of Law \& Slepecky (1961) and of Poindexter \& Eley (1983): cells from a $5 \mathrm{ml}$ culture sample were collected by centrifugation $\left(8000 \mathrm{~g}, 10 \mathrm{~min}, 4^{\circ} \mathrm{C}\right)$, and washed with $10 \mathrm{~mm}$-Tris/ $\mathrm{HCl} / \mathrm{saline}(\mathrm{NaCl}$ $0.9 \%, \mathrm{w} / \mathrm{v}$ ) buffer $\mathrm{pH} 7 \cdot 0$. The pellet was suspended in $5 \mathrm{ml} 0.5 \%(\mathrm{w} / \mathrm{v})$ sodium hypochlorite and left for $1 \mathrm{~h}$ at $37^{\circ} \mathrm{C}$ to allow total lysis of the cells. The PHB granules were then collected by centrifugation $(4000 \mathrm{~g}$, $10 \mathrm{~min}, 4^{\circ} \mathrm{C}$ ), washed successively in distilled water, acetone and ethanol, and finally dissolved in hot chloroform. After the chloroforminsoluble part had been discarded, the chloroform was evaporated and the residue was hydrolysed and dehydrated with concentrated sulphuric acid to obtain crotonic acid, which can be quantified by its absorbance at $235 \mathrm{~nm}$, according to Zevenhuizen (1974). A complete absorbance spectrum was measured for each sample using sulphuric acid as a blank

Pyruvate determination. Pyruvate concentration in the culture supernatant was measured by spectrophotometric analysis of NADH oxidation at $340 \mathrm{~nm}$ in the presence of lactate dehydrogenase (Czok \& Lamprecht, 1974). This method permits detection of pyruvate concentrations as low as $3 \mu \mathrm{M}$.

Determination of respiratory activities. Cells were washed by centrifu-

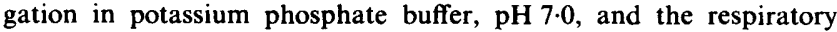
activities were determined polarographically at $28^{\circ} \mathrm{C}$ using a Clarktype oxygen electrode (Hansatech, $\mathrm{CBH} 2 \mathrm{Box}$, adjustable volume) with a chart recorder. Oxygen concentration in the experimental system was calibrated using the method of Robinson \& Cooper (1970) and the respiratory activities were determined as previously described (Beffa $e t$ al., 1988). The reaction mixture ( $1 \mathrm{ml}$ final vol.) contained only the mineral basal medium for the determination of endogenous respiratory activity, and the same medium supplemented with $25 \mathrm{~mm}$-pyruvate (final concentration) for the measurement of total respiratory activity. The measurements were performed with 0.2 to $0.4 \mathrm{mg}$ dry wt cells ml ${ }^{-1}$ and are expressed in $\mathrm{nmol} \mathrm{O}_{2}$ consumed ( $\mathrm{ml}$ culture $)^{-1} \mathrm{~min}^{-1}$.

Other methods. Total polyphosphates (acid soluble plus insoluble) were determined from $5 \mathrm{ml}$ of washed cells according to Poindexter \& Eley (1983) and Clark et al. (1986). Dry weight was measured after drying washed cells for $48 \mathrm{~h}$ at $100^{\circ} \mathrm{C}$. Total protein was estimated with the bicinchoninic acid method (P. K. Smith et al., 1985). Non-linear fitting of the models to the experimental data was performed by standard algorithms of minimization.

\section{Results}

The term 'exponential' will be restricted to qualify unlimited balanced growth at a constant rate. It will never be used for exogeneously limited growth at steady state. The subscript 'exp' will therefore qualify all parameters related to this kind of growth, such as the exponential growth rate $\mu_{\exp }$ (often referred to as $\mu_{\max }$ ).

\section{Observation of transitions}

Similar experiments were undertaken in both fermenter installations. Each continuous culture was inoculated with an exponentially growing preculture of $A$. autotrophicum on pyruvate. After an initial exponential growth phase at rate $\mu_{\exp } \approx 0.32 \mathrm{~h}^{-1}$ (see below, Fig. 3), the culture became limited in pyruvate and relaxed to a pyruvate-limited steady state. Then a given volume of concentrated pyruvate solution was injected into the culture. The volume added had a negligible influence, except on the pyruvate concentration. The growth dynamics changed immediately, turning from steady state to apparently linear growth (Fig. 1). Despite the fact that the characteristic exponential curvature is only faintly marked on an hour scale, the precision of biomass measurements was sufficient to reveal the absence of any positive curvature. This typical growth response appeared in all experiments for dilution rates ranging from 0.049 to $0.246 \mathrm{~h}^{-1}$, lasted for at least $1 \mathrm{~h}$ and was observed for up to $3 \mathrm{~h}$ depending on the injected volume and dilution rate. The pyruvate pulse and the reappearance of pyruvate limitation were both correlated with sudden changes in $\mathrm{pH}$ and oxygen signals. The aspect of linear growth termination depended on the particular experimental conditions. It was either sudden and correlated with the reappearance of pyruvate limitation for all transitions lasting less than $2 \mathrm{~h}$, or progressive, before the reappearance of limitation (see below, Fig. 4). Finally, each culture relaxed back to its previously observed steady state.

The intersection of the regression lines fitting steady state and linear growth is located very close to the injection time. This was observed in all experiments and is fully correlated both with sudden changes in dissolved oxygen and in $\mathrm{pH}$ values. Experimental conditions do not permit the demonstration of the simultaneity of the onset of linear growth and pyruvate injection with an accuracy of more than $30 \mathrm{~s}$. Changes in oxygen concentration on the other hand, can be determined in a matter of seconds.

\section{Modifications of cell composition}

Table 1 summarizes some relevant measurements made during a linear growth response. Only negligible PHB concentrations were detected in cultures at steady state, whereas PHB accumulated linearly during the transitions (Fig. 2). During the linear growth response, small 


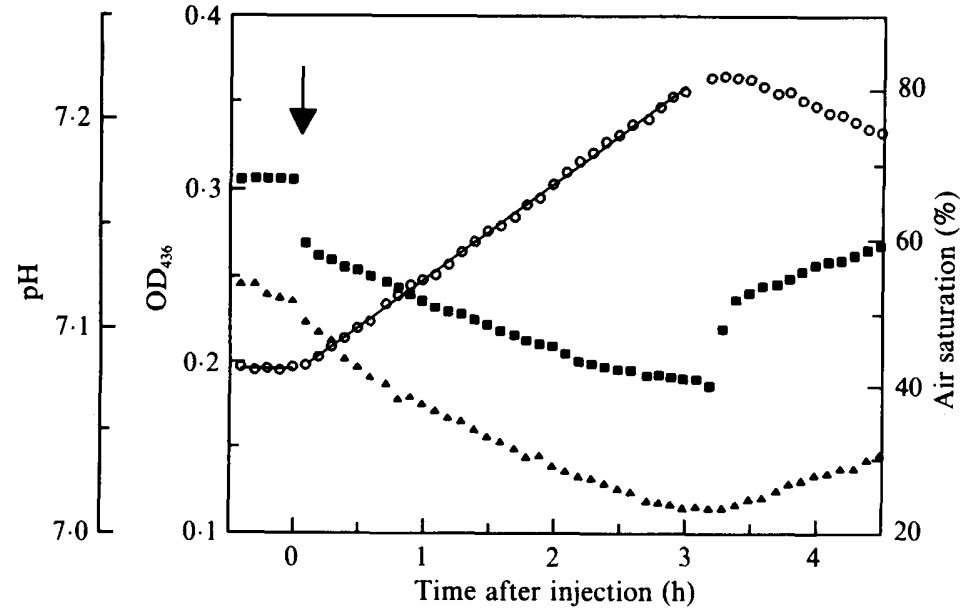

Fig. 1. Characteristic linear growth response after the injection (arrow) of $2 \mathrm{ml}$ pyruvate ( $3 \mathrm{M}$ ) into a 0.81 pyruvate-limited culture at steady state $\left(D=0.160 \mathrm{~h}^{-1}, S_{\mathrm{f}}=7.5 \mathrm{~mm}\right) . \mathrm{O}, \mathrm{OD}_{436}$ with $2 \mathrm{~mm}$ optical path; $\boldsymbol{\Delta}, \mathrm{pH} ; \boldsymbol{\square}$, percentage air saturation.

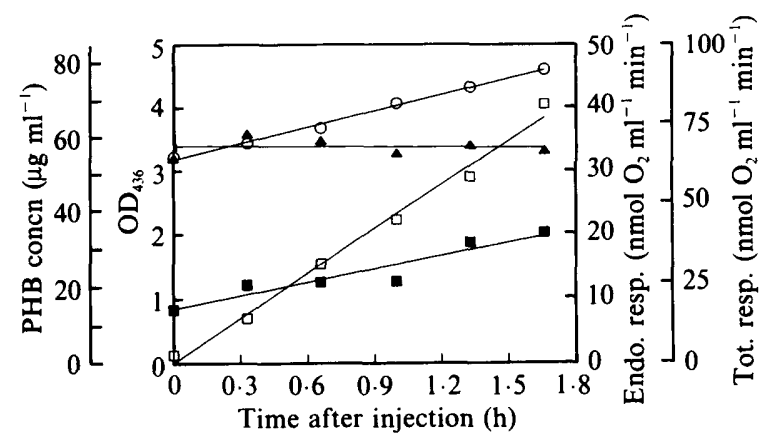

Fig. 2. Production of PHB ( $\square), \mathrm{OD}_{436}$ with $1 \mathrm{~cm}$ optical path $(O)$, endogenous respiration $(\mathbb{\square})$ and total respiration $(\boldsymbol{\Lambda})$ after the injection of $15 \mathrm{ml}$ pyruvate $(3 \mathrm{M})$ into a 3.41 pyruvate-limited culture at steady state $\left(D=0.134 \mathrm{~h}^{-1}, S_{\mathrm{f}}=22.26 \mathrm{mM}\right)$. inclusions, probably of $\mathrm{PHB}$, were observed in cells under phase-contrast microscopy, whereas these inclusions were not visible in cells from cultures at steady state. The ratio of dry weight to optical density appeared almost constant, with or without the presence of PHB inclusions, but this apparent constancy could be restricted to PHB contents of less than $10 \%(w / w)$. Correlated with PHB synthesis, the endogenous respiration rate increased while the total respiration rate showed no significant variation. The total polyphosphate content during the transitions remained below the detection limit of the method used. Polyphosphate accumulation does not seem to play any role here. However, volutin inclusions have been observed in $A$. autotrophicum under other growth conditions (WalterMauruschat et al., 1977).

Table 1. Measurement of relevant physiological parameters after a pyruvate pulse

Pyruvate $(15 \mathrm{ml} ; 3 \mathrm{M})$ was injected into a 3.41 culture at steady state $\left(D=0.134 \mathrm{~h}^{-1}\right.$, $S_{\mathrm{f}}=22.26 \mathrm{mM}$, Biostat fermenter). Values at $0 \mathrm{~h}$ correspond to the steady state.

\begin{tabular}{llccc}
\hline \hline & & \multicolumn{3}{c}{ Time after injection (h): } \\
\cline { 3 - 5 } Measurement & \multicolumn{1}{c}{ Unit } & 0 & 1 & 2 \\
\hline Turbidity & $\mathrm{OD}_{436}(1 \mathrm{~cm}$ optical path $)$ & 3.197 & 4.017 & 4.894 \\
Dry wt & $\mathrm{mg} \mathrm{ml}^{-1}$ & 0.670 & 0.845 & 1.019 \\
Turbidity/dry wt & $\mathrm{OD} \mathrm{mg}^{-1}$ & $4 \cdot 77$ & $4 \cdot 75$ & 4.80 \\
PHB & $\mu \mathrm{g} \mathrm{ml}^{-1}$ & $<0.2$ & 37.5 & 69.2 \\
PHB/dry wt & $\% \mathrm{mg} \mathrm{mg}^{-1}$ & $<0.03$ & 4.4 & 6.8 \\
Protein & $\mathrm{mg} \mathrm{ml}^{-1}$ & 0.50 & 0.57 & 0.64 \\
Protein/dry wt & $\% \mathrm{mg} \mathrm{mg}^{-1}$ & 75 & 67 & 63 \\
Polyphosphate & $\mu \mathrm{g} \mathrm{ml}^{-1}$ & $<0.2$ & $<0.2$ & $<0.2$ \\
Endo. resp. & $\mathrm{nmol} \mathrm{O}_{2} \mathrm{ml}^{-1} \mathrm{~min}^{-1}$ & 8.4 & 12.6 & 21.3 \\
Tot. resp. & $\mathrm{nmol} \mathrm{O}_{2} \mathrm{ml}^{-1} \mathrm{~min}^{-1}$ & 64.1 & 65.5 & 70.4 \\
\hline \hline
\end{tabular}


Table 2. Determination of $q_{\exp }$ at the start-up of an experiment with the Biostat fermenter and determination of $q_{\mathrm{lin}}^{\mathrm{o}}$ later in the same experiment after a pyruvate pulse

Pyruvate $(15 \mathrm{ml} ; 3 \mathrm{M})$ was injected into a 3.41 culture at steady state $\left(D=0.134 \mathrm{~h}^{-1}\right.$,
$S_{\mathrm{f}}=22.26 \mathrm{mM}$. The fixed values were obtained from culture conditions and from
biomass growth kinetics. The fitted values were computed on the pyruvate
disappearance kinetics.

\section{Kinetics of substrate disappearance}

During steady state, the residual pyruvate concentration was undetectable by the assay used, so the affinity constant of $\boldsymbol{A}$. autotrophicum for pyruvate is certainly within the micromolar range. In turn, during exponential or linear growth, pyruvate concentrations in the millimolar range were recorded. As a consequence, it was postulated that the pyruvate uptake system(s) was (were) saturated during both types of growth.

During start-up exponential growth in continuous culture, the evolution of the substrate concentration $S_{\text {exp }}$ is described by the following differential equation

$$
\frac{\mathrm{d} S_{\text {exp }}}{\mathrm{d} t}=\left(S_{\mathrm{f}}-S_{\text {exp }}\right) D-q_{\text {exp }} X_{0} \mathrm{e}^{\left(\mu_{\text {exp }}-D\right) t}
$$

where $\mu_{\text {exp }}$ is the exponential growth rate, $D$ the dilution rate, $S_{\mathrm{f}}$ the substrate concentration in the feed, $q_{\text {exp }}$ the constant substrate consumption rate per biomass unit and $X_{0}$ the initial biomass. This differential equation can be resolved into

$$
S_{\text {exp }}(t)=S_{\mathrm{f}}+\mathrm{e}^{-D t}\left[S_{0}-S_{\mathrm{f}}+\frac{X_{0} q_{\exp }}{\mu_{\exp }}\left(1-\mathrm{e}^{\mu_{\text {exp }}}\right)\right]
$$

with $S_{0}$ as the initial substrate concentration.

Linear growth is biomass accumulation at a constant rate, because a linear function of time is the primitive of a constant function. A simple hypothesis for linear growth could involve a constant consumption rate for the whole culture and a constant growth yield. Then, the following equation would describe the substrate-disappearance kinetics during linear growth

$$
\frac{\mathrm{d} S_{\text {lin }}}{\mathrm{d} t}=\left(S_{\mathrm{f}}-S_{\text {lin }}\right) D-Q_{\text {lin }}^{\text {whole }}
$$

where $Q_{\text {lin }}^{\text {whole }}$ is the constant consumption rate by volume unit of the whole culture. In fact, the physiology underlying this hypothesis is more complicated than it appears, because of the consumption rate per biomass unit, which has to decrease with time, and because of the ambiguous meaning of a constant growth yield during a growth which is known to be unbalanced. Formally, a consumption rate per biomass unit, $q_{\mathrm{lin}}^{0}$, can be defined at the beginning of linear growth when the biomass is equal to the steady state biomass $X_{\text {SS }}$

$$
q_{\text {lin }}^{0}=\frac{Q_{\text {lin }}^{\text {whole }}}{X_{\mathrm{SS}}}
$$

so the differential equation (3) can be rewritten

$$
\frac{\mathrm{d} S_{\mathrm{lin}}}{\mathrm{d} t}=\left(S_{\mathrm{f}}-S_{\mathrm{lin}}\right) D-q_{\mathrm{lin}}^{0} X_{\mathrm{sS}}
$$

and admits the solution

$$
S_{\mathrm{lin}}(t)=S_{\mathrm{f}}-\frac{q_{\mathrm{lin}}^{0} X_{\mathrm{SS}}}{D}+\mathrm{e}^{-D t}\left(S_{0}-S_{\mathrm{f}}+\frac{q_{\mathrm{lin}}^{0} X_{\mathrm{SS}}}{D}\right)
$$

$S_{\text {exp }}(t)$ and $S_{\text {lin }}(t)$ can easily be distinguished graphically, because of the negative curvature of $S_{\text {exp }}(t)$

$$
\begin{aligned}
\frac{\mathrm{d}^{2} S_{\text {exp }}}{\mathrm{d} t^{2}}=- & \left(S_{\mathrm{f}}-S_{\exp }\right) D^{2}-q_{\exp } X_{0}\left(\mu_{\exp }-2 D\right) . \\
& \mathrm{e}^{\left(\mu_{\text {exp }}-D\right) t}<0 \forall t, t \geq 0 \\
& \text { if } S_{\text {exp }}(0)<S_{\mathrm{f}}+q_{\exp } X_{0} \frac{2 D-\mu_{\text {exp }}}{D^{2}}
\end{aligned}
$$




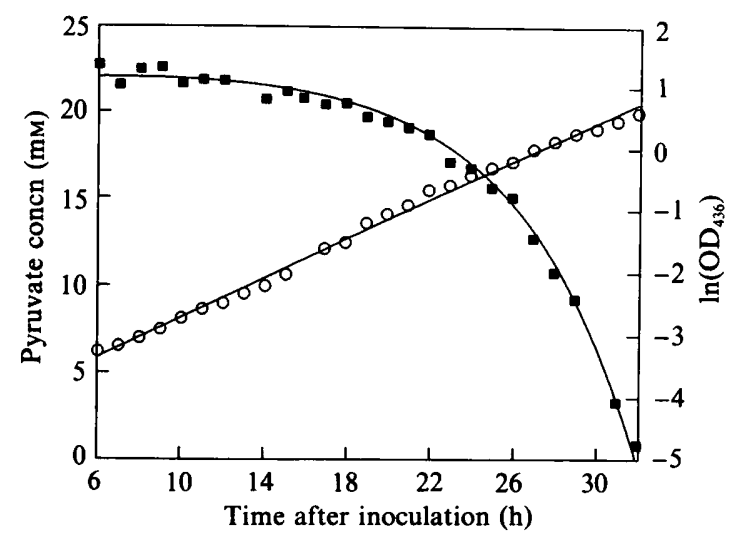

Fig. 3.

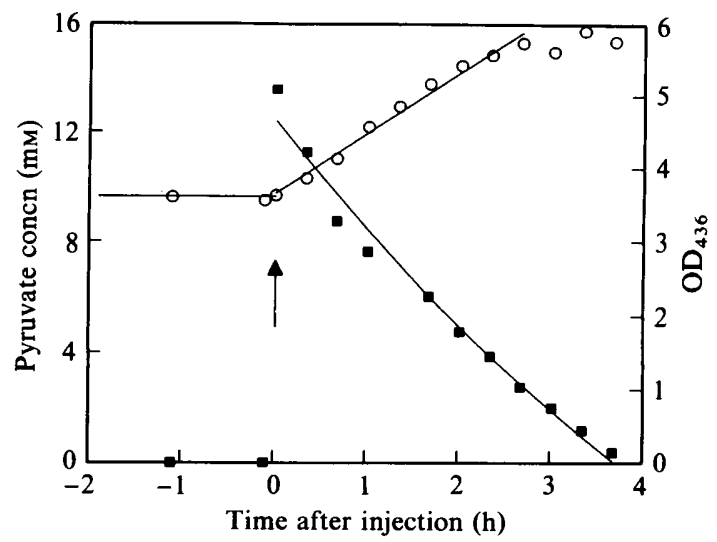

Fig. 4.

Fig. 3. $\ln \left(\mathrm{OD}_{436}\right)$ with $1 \mathrm{~cm}$ optical path $(O)$ and pyruvate concentration $(\square)$ during the exponential growth at start-up of a continuous culture $\left(D=0.134 \mathrm{~h}^{-1}, S_{\mathrm{f}}=22.26 \mathrm{mM}\right)$. Data fitted with model $S_{\text {exp }}(t)$ (eq. 2).

Fig. 4. $\mathrm{OD}_{436}$ with $1 \mathrm{~cm}$ optical path $(\mathrm{O})$ and pyruvate concentration $(\square)$ before and after the injection (arrow) of $15 \mathrm{ml}$ pyruvate $(3 \mathrm{M}$ ) into a 3.41 pyruvate-limited culture at steady state $\left(D=0.134 \mathrm{~h}^{-1}, S_{\mathrm{f}}=22.26 \mathrm{~mm}\right)$. Data fitted with model $S_{\text {lin }}(t)(\mathrm{eq} .6)$.

prevailing at least at the start-up of the culture $\left[S_{\text {exp }}(0)\right.$ close to $S_{\mathrm{f}}$ and $X_{0}$ small] against the slight positive curvature of $S_{\text {lin }}(t)$

$$
\frac{\mathrm{d}^{2} S_{\text {lin }}}{\mathrm{d} t^{2}}=D\left[Q_{\text {lin }}^{\text {whole }}-D\left(S_{\mathrm{f}}-S_{\text {lin }}\right)\right]>0 \text { as } Q_{\text {lin }}^{\text {whole }}>D S_{\mathrm{f}}
$$

Kinetic data of pyruvate disappearance were recorded during both exponential and linear growth and both models were fitted to the data. The values obtained for the parameters are shown in Table 2, and the fits of the models to experimental data are presented in Figs 3 and 4. The hypotheses underlying the two sets of substrateconsumption kinetics appeared to be satisfied for the respective growth behaviours. Cross-testing of the models did not yield acceptable fits because of the opposing curvatures. Fig. 4 shows a case where linear growth ended before pyruvate had become limiting again. Nevertheless, the hypothesis of a constant consumption rate for the whole culture still appeared valid up to the reappearance of pyruvate-limitation. The two consumption rates per biomass unit are strikingly close

$$
\begin{aligned}
& q_{\exp }=1.75 \mathrm{mmol} \mathrm{OD}^{-1} \mathrm{~h}^{-1} \text { and } \\
& q_{\mathrm{lin}}^{0}=1.76 \mathrm{mmol} \mathrm{OD}^{-1} \mathrm{~h}^{-1}
\end{aligned}
$$

The growth at steady state seems to have only a negligible influence on the constancy of the (maximal) uptake rate of the saturated consumption system. This apparent constancy was verified by the data from the automated turbidity-measuring system. Admitting the same substrate disappearance kinetics, the solution equations, $S_{\text {exp }}(t)$ and $S_{\text {lin }}(t)$, were rearranged in order to relate the consumption rates with the time required for substrate disappearance. The respective consumption rate was then evaluated for each pulse experiment. The constancy of the maximal potential consumption rate of pyruvate was roughly confirmed in the range of observed dilution rates $\left(0.049\right.$ to $\left.0.246 \mathrm{~h}^{-1}\right)$.

\section{Effects of dilution rate}

The steady state optical density, $X_{\mathrm{SS}}$, and the slope, $\Delta X / \Delta \mathrm{d} t$, of the linear response were recorded at different dilution rates in the parallel fermenter installation in order to compute the initial linear growth rate

$$
\mu_{\mathrm{lin}}^{0}=\frac{1}{X_{\mathrm{ss}}} \frac{\Delta X}{\Delta t}+D
$$

This initial linear growth rate was taken into account instead of the slope alone, because while the potential maximal consumption rate appeared constant, the steady state biomass varied according to the dependence of growth yield on dilution rate (Pirt 1965; Esener et al., 1983). Fig. 5 shows the $\mu_{\text {lin }}^{0}$ distribution against the dilution rate. A negative regression correlates both parameters satisfactorily

$$
\mu_{\text {lin }}^{0}=0.55-0.61 D
$$

showing that the initial linear growth rate is always greater than the exponential growth rate $\left(\mu_{\exp }=0.32 \mathrm{~h}^{-1}\right)$ at any observed dilution rate. Biomass linear overproduction is proportional to the difference between the conditioning dilution rate and the maximal growth rate, or, from another point of view, to the sudden overconsumption of substrate. Moreover, the intercept of the regression line with $\mu_{\text {exp }}$ is close to the washout dilution 


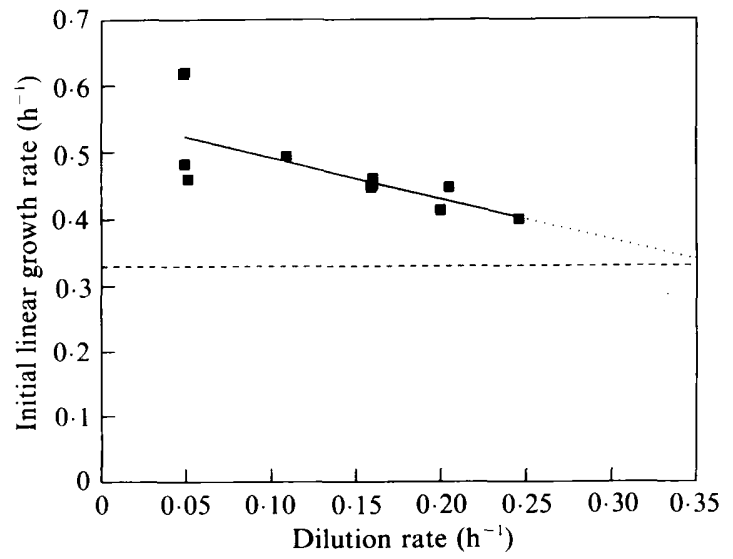

Fig. 5. Initial linear growth rate, $\mu_{\text {lin }}^{0}$, plotted against the dilution rate (ם) for ten pulse experiments computed from data obtained with the small fermenters (example shown in Fig. 1). _ , Regression line; -- , (non-limited) exponential growth rate.

rate; this implies that no linear response would be expected if extra substrate were injected into an already (non-limited) exponentially growing culture. Therefore, in these cells PHB accumulation is not to be expected in significant amounts, just as was observed.

\section{Discussion}

\section{Pyruvate as sole limiting factor}

It was assumed that pyruvate was the sole limiting factor at steady state according to the following experimental evidence. (a) Steady-state pyruvate concentration is not detectable for any tested feed concentration. (b) Schlegel's medium allows $A$. autotrophicum to grow up to $2.5 \mathrm{mg}$ dry $\mathrm{wt} \mathrm{ml}^{-1}$, twice the highest biomass recorded here; ammonium then becomes limiting, provided carbon and energy are in excess (Fasnacht 1988, and personal observations). (c) After several successive pulse experiments, the pyruvate feed concentration was increased; the culture then grew up to a new steady state with a biomass concentration higher than recorded during the pulse experiments. $(d)$ The characteristic linear response was observed under various dissolved oxygen partial pressures ranging from 16 to $60 \%$ air saturation, depending on air inflow rates, while oxygen limitation was observed only below $3 \%$ air saturation in other experiments. (e) The characteristic transitions were similar in both basal and half-phosphate concentration media.

\section{Linear growth kinetics}

The growth kinetics following a pyruvate pulse are clearly non-exponential: the initial transient growth rate is distinctly higher than the exponential one (Fig. 5). This growth is unbalanced, as revealed by the accumulation of
PHB and by the decrease in the protein fraction of the biomass (Table 1). PHB accumulation is related to an increase in endogenous respiration. However, PHB does not account for the total increase in biomass: in $2 \mathrm{~h}$, the dry biomass increased by $349 \mu \mathrm{g} \mathrm{ml}^{-1}$, whereas only $69 \cdot 2 \mu \mathrm{g}$ PHB ml ${ }^{-1}$ accumulated. Simultaneously, the protein content of the culture increased by $140 \mu \mathrm{g} \mathrm{ml}^{-1}$. Clearly, both phenomena (PHB and protein synthesis) participate in the biomass increase.

Despite the complexity of such unbalanced growth, a simple linear growth model for total biomass suffices to describe the observed kinetics of growth and substrate disappearance. It should therefore be considered as a useful local description. Its adequacy is certainly restricted to the accuracy of the measurements which is probably not the case for true exponential growth. The effect of the dilution rate, which should give a slight negative curvature to the kinetics of biomass accumulation at a constant rate, is actually negligible.

In his work on growth kinetics of single cells or synchronized cultures of Escherichia coli, Kubitschek (1990) has proposed a linear growth model as a general model for dry weight increase during one cell cycle. The macromolecular (cytoplasmic) fraction accumulates exponentially (Ecker \& Kokaisl, 1969), but as shown by Kubitschek \& Pai (1988) the precursor-pool sizes vary with a maximum at mid-cycle and a minimum at division time, so the total weight increase, macromolecules plus precursors, remains linear. This linear growth pattern is explained by the constancy of uptake during the cell cycle, the proteins responsible for consumption (periplasmic binding-proteins and membrane-bound proteins) being synthesized only near division time (reviewed in Kubitschek, 1990). The linear biomass increase and the constant substrate consumption-rate for the whole culture are consistent with our observations during the pulse experiments. Therefore, pulsing the culture with the limiting factor appears to induce a coordinated behaviour of the cell population. In $A$. autotrophicum, cell numbers are poorly correlated to biomass parameters during transient growth, which is not surprising for an elongated organism subject to variation in cell length. Thus we could not introduce with sufficient accuracy the cell numbers as a parameter for a short $(2 \mathrm{~h})$ period. Even if our results cannot be used as experimental evidence for Kubitschek's model, this model does allow prediction of biomass behaviour consistent with our observations.

\section{Role of $P H B$}

The role traditionally attributed to PHB is that of a reserve material, which is synthesized during growth limited by a non-carbon compound (reviewed in Ander- 
son \& Dawes, 1990). Senior \& Dawes (1971) have also proposed that PHB can be viewed as a sink of reducing power. However, it has to be pointed out that during our pulse experiments, PHB was shown to be synthesized, although in small amounts, under a complete lack of exogenous limitation. This accumulation was apparently linear (Fig. 2). Such behaviour has to be considered in the light of other similar delimitation experiments. I. W. Smith et al. (1954) showed that Klebsiella aerogenes transferred from a phosphate-limiting to a non-limiting medium transiently synthesized polyphosphate inclusions. With a glucose-limited continuous culture of the same organism, Harisson \& Maitra (1969) showed that a pulse of glucose causes a transient excretion of pyruvate and another unidentified substance. Moreover, one of their figures presents a transient linear excess production of $\mathrm{CO}_{2}$ and slightly positively curved glucose disappearance kinetics; a linear growth pattern can be suspected from these data. A methanol pulse on a methanol-limited continuous culture of a thermophilic Bacillus strain resulted in a rapid excretion of formaldehyde into the medium (Brooke et al., 1989); despite the subsequent autopoisoning of the cells, formaldehyde accumulation appeared strikingly linear. Excretion or accumulation of 'byproducts' (polyphosphate, pyruvate, formaldehyde or PHB) during unbalanced growth is certainly of major importance for actual survival, because the cells are temporarily incapable of metabolizing all the consumed substrate. Importance of overflow metabolism for cell survival can be compared with the need for efficient uptake-regulation in yeasts: with a mutant of Saccharomyces cerevisiae defective for maltose uptake-regulation, Entian \& Loureiro-Dias (1990) showed that a maltose pulse results in cell autopoisoning caused by intracellular glucose accumulation. The fact that exponential growth metabolism cannot be achieved instantaneously by a previously slow-growing culture is underlined here by the governing role of dilution rate in culture conditioning (Fig. 5). Many different hypotheses can be formulated in order to identify the nature of the bottleneck involved, ribosome concentration or nitrogen-uptake regulation, for example, admitting such a bottleneck really does exist. But more generally, as the macromolecular composition depends on the growth rate (discussed by Esener et al., 1982, and Wanner \& Egli, 1990), a certain lag-time is undoubtedly necessary to adapt the macromolecular composition to the new environmental and growth conditions.

A. autotrophicum temporarily 'wastes' the over-consumed substrate by synthesizing PHB, which could be considered as a sink protecting the cell against a transient metabolic overflow. The classical role of PHB as a 'strategic' reserve for future survival remains of first ecological significance, but it is secondary for actual cell survival.
The research reported here was supported by grant 3.645-87 of the Swiss National Science Foundation. We are grateful to Monica Fischer and Catherine Fischer who corrected the manuscript and to Sebastien David for his technical assistance.

\section{References}

Anderson, A. J. \& DAwes, E. A. (1990). Occurrence, metabolism, metabolic role and industrial uses of bacterial polyhydroxyalcanoates. Microbiological Reviews 54, 450-472.

ARAGNo, M. \& SChlegel, H. G. (1978a). Aquaspirillum autotrophicum, a new species of hydrogen-oxidizing, facultatively autotrophic bacteria. International Journal of Systematic Bacteriology 28, 112-116.

ARAgno, M. \& SCHLegel, H. G. $(1978 b)$. Physiological characterization of the hydrogen bacteria Aquaspirillum autotrophicum. Archives of Microbiology 116, 221-229.

ARAGNO, M. \& SCHLEGEL, H. G. (1981). The hydrogen-oxidizing bacteria. In The Prokaryotes: a Handbook on Habitats, Isolation and Identification of Bacteria, pp. 865-893. Edited by M. P. Starr, H. Stolp, H. G. Trüper, A. Balows \& H. G. Schlegel. Berlin/Heidelberg/ New York: Springer-Verlag.

Beffa, T., Pezet, R. \& Turian, G. (1988). Endogenous elemental sulfur production from L-cysteine in dormant $\alpha$-spore of Phomopsis viticola. Current Microbiology 17, 259-263.

Brooke, A. G., Watling, E. M., Atrwood, M. M. \& Tempest, D. W (1989). Environmental control of metabolic fluxes in thermotolerant methylotrophic Bacillus strains. Archives of Microbiology 151, 268-273.

Clark, J. E., Beegen, H. \& WoOd, H. G. (1986). Isolation of intact chains of polyphosphate from "Propionibacterium shermanii" grown on glucose or lactate. Journal of Bacteriology 168, 1212-1219.

Czox, R. \& LAmprecht, W. (1974). Pyruvate, phosphoenolpyruvate and D-glycerate-2-phosphate. In Methods of Enzymatic Analysis, vol. 3, pp. 1446-1451. Edited by H. U. Bergmeyer. New York: Academic Press.

ECKER, R. E. \& KOKAISL, G. (1969). Synthesis of protein, ribonucleic acid, and ribosomes by individual bacterial cells in balanced growth. Journal of Bacteriology 3, 1219-1226.

ENTIAN, K.-D. \& Loureiro-Dias, M. C. (1990). Misregulation of maltose uptake in a glucose repression defective mutant of Saccharomyces cerevisiae leads to glucose poisoning. Journal of General Microbiology 136, 855-860.

Esener, A. A., Veerman, T., Roels, J. A. \& Kossen, N. W. F. (1982). Modeling of bacterial growth; formulation and evaluation of a structured model. Biotechnology and Bioengineering 24, 1749-1764.

EsEner, A. A., Roels J. A. \& Kossen, N. W. F. (1983). Theory and applications of unstructured growth models: kinetic and energetics aspects. Biotechnology and Bioengineering 25, 2803-2841.

FASNACHT, M. (1988). Étude physiologique de la mixotrophie chez l'hydrogénobacterie Aquaspirillum autotrophicum. PhD thesis, Université de Neuchâtel, Switzerland.

Harisson, D. E. F. \& MaITra, P. K. (1969). Control of respiration and metabolism in growing Klebsiella aerogenes: the role of adenine nucleotides. Biochemical Journal 112, 647-656.

KUBITSCHEK, H. E. (1990). Cell growth and abrupt doubling of membrane proteins in Escherichia coli during the division cycle. Journal of General Microbiology 136, 599-606.

KUBITSCHEK, H. E. \& PaI, S. R. (1988). Variation in precursor pool size during the division cycle of Escherichia coli: further evidence for linear cell growth. Journal of Bacteriology 170, 431-435.

LAW, J. H. \& SLEPECKY, R. A. (1961). Assay of poly- $\beta$-hydroxybutyric acid. Journal of Bacteriology 82, 33-36.

PIRT, S. J. (1965). The maintenance energy of bacteria in growing cultures. Proceeding of the Royal Society London 163B, 224-231.

PoIndeXter, J. S. \& EleY, L. F. (1983). Combined procedure for assay of poly- $\beta$-hydroxybutyric acid and inorganic polyphosphate. Journal of Microbiological Methods 1, 1-17.

Robinson, J. \& CoOPER, J. M. (1970). Method of determining oxygen concentration in biological media, suitable for calibration of the oxygen electrode. Analytical Biochemistry 33, 390-399. 
Schlegel, H. G., Kaltwasser, H. \& Gottschalk, G. (1961). Ein submersverfahren zur Kultur wasserstoffoxydierender Bacterien: Wachstumsphysiologische Untersuchungen. Archives of Microbiology 38, 209-222.

Senior, P. J. \& Dawes, E. A. (1971). Poly- $\beta$-hydroxybutyrate biosynthesis and the regulation of glucose metabolism in Azotobacter beijerinckii. Biochemical Journal 125, 55-66.

Smith, I. W., Wilkinson, J. F. \& Duguid, J. P. (1954). Volutin production in Aerobacter aerogenes due to nutrient imbalance. Journal of Bacteriology 68, 450-463.

Smith, P. K., Krohn, R. I., Hermanson, G. T., Mallia, A. K., Gartner, F. H., Provenzano, M. D., Fujmoto, E. K., Goeke,
N. M., Olson, B. J. \& KLENK, D. C. (1985). Measurement of protein using bicinchoninic acid. Analytical Biochemistry 150, 76-85.

Walter-Mauruschat, A., Aragno, M., Mayer, M. \& Schlegel, H. G. (1977). Micromorphology of Gram-negative hydrogen bacteria. II. Cell envelope, membranes and cytoplasmic inclusions. Archives of Microbiology 114, 101-110.

WANNER, U. \& EGLI, T. (1990). Dynamics of microbial growth and cell composition in batch culture. FEMS Microbiology Reviews 75, 19-44.

Zevenhuizen, L. P. T. M. (1974). Spectrophotometric assay of longchain unsaturated and hydroxy fatty acids in concentrated sulfuric acid. Analytical Biochemistry 58, 146-154. 